\title{
Costos directos de varicela complicada en una población pediátrica de Colombia
}

\section{Direct costs of complicated chicken pox in a Colombian pediatric population}

\author{
Nelson Alvis-Guzmán ${ }^{1}$, Angel Paternina-Caicedo ${ }^{1}$, Luis Alvis-Estrada ${ }^{1}$ y \\ Fernando De la Hoz-Restrepo ${ }^{2}$
}

\footnotetext{
1 Grupo de Investigación en Economía de la Salud. Facultad de Ciencias Económicas, Universidad de Cartagena. Cartagena de Indias, Colombia.nalvis@yahoo.com, angel.paternina@gmail.com, alvisluis@ gmail.com.

2 Departamento de Salud Pública. Universidad Nacional de Colombia. Bogotá. fpdelahozr@bt.unal.edu.co.
}

Recibido 20 Marzo 2011/Enviado para Modificación 7 Diciembre 2011/Aceptado 22 Diciembre 2011

\section{RESUMEN}

Objetivo Estimar los costos directos de varicela en una población pediátrica de Colombia.

Método Se diseño un estudio de casos retrospectivo sobre todos los casos de varicela diagnosticados de 2005-2008 en el Hospital Infantil Napoleón Franco Pareja de Cartagena de Indias, Colombia. Fue utilizada la perspectiva del hospital. Se buscaron costos de atención, laboratorio, imagenología y medicamentos. EI microcosteo se realizó en pesos colombianos del 2010. Se realizó un ajuste por inflación.

Resultados La mediana de costos totales hospitalarios fue de \$ 898766 (Q1: \$ 197 348; Q3: \$ 1195 262). La mediana de costo por día hospitalario fue de \$ 221 777 (Q1: \$ 97 027; Q3: \$ 293 740). En menores de 1 año la mediana de costo fue de \$ 980742 (Q1: \$ 905 708; Q3: \$ 1026 031). En pacientes de 5-12 años la mediana de costo fue de \$ 105833 (Q1: \$ 39 568; Q3: \$ 891 824).

Conclusiones. Los resultados se asemejan con estudios previos sobre el tema (en Panamá, e incluso algunos países desarrollados), evidenciando un relativo alto costo de enfermedad por varicela en Colombia. Estos resultados aumentan la evidencia a favor de la vacunación, e invitan a decisores en salud en Colombia a considerar la introducción de la vacunación contra varicela.

Palabras Clave: Varicela, costos y análisis de costo; vacuna contra la varicela (fuente: DeCS, BIREME).

\section{ABSTRACT}

Objective Estimating the cost of chicken pox in a Colombian pediatric population. Methodology This was a retrospective case study which searched for all diagnosed 
chicken pox cases in the Napoleón Franco Pareja children's hospital (Cartagena, Colombia), during 2005-2008. The hospital's records/perspective was used. Cost related to health personnel, lab, diagnostic images and drugs were searched. The micro-costing was made at Colombian peso prices for 2010. An adjustment was made for inflation.

Results Mean hospital costs were \$ 898,766 (Q1: \$ 197,348; Q3: \$ 1,195,262). Mean hospital cost per day was \$221,777 (Q1: \$ 97,027; Q3: \$ 293,740). Mean cost <1 year-old patients was $\$ 980,742$ (Q1: \$ 905,708; Q3: \$1,026,031). Mean cost was \$105,833 in 5-12 year-old patients (Q1: \$ 39,568; Q3: \$ 891,824).

Conclusions The results were similar to those of previous studies (in Panama and some developed countries) highlighting relatively high illness costs in Colombia. These results increase the evidence in favor of vaccination and invite Colombian public health officials to consider introducing a chicken pox vaccine into Colombia.

Key Words: Chickenpox, costs and cost analysis, chickenpox vaccine (source: $\mathrm{MeSH}, \mathrm{NLM}$ ).

$\mathrm{L}$ a varicela es una enfermedad infecciosa de predominio infantil causada por el Herpes virus Varicela Zoster. Es altamente contagiosa en la población, y a pesar del subregistro del $95 \%$ de los casos no complicados, se reportó en Colombia según Espinal (1998) una incidencia anual de 101 por 100000 habitantes en 1996 (1). El 62\% de estos casos se reportó en niños de 0 a 14 años.

La incidencia de varicela en otros países es mucho más alta que la reportada en Colombia, con 1749 casos de varicela en 1998, Italia tiene una incidencia de casos complicados y no complicados de 5,2 \% de toda su cohorte de niños por año (2). Los costos derivados de esta enfermedad en la sociedad pueden llegar a ser elevados, especialmente por la pérdida de productividad en los padres o cuidadores, y los casos complicados de esta enfermedad.

Un estudio en Panamá mostró que la media de costo por paciente con varicela es US\$1 209. Evidenciando además, una alta tasa de complicaciones en los niños hospitalizados con varicela adquirida en la comunidad (92\%) (3), lo cual hace de esta patología relativamente benigna, una enfermedad costosa, con potenciales implicaciones en el sistema de salud de países que no hayan implementado la vacunación.

Teniendo esto en cuenta, y como estimación inicial para la posterior evaluación del costo-efectividad de la vacunación universal de varicela en 
la población pediátrica, se realizó una estimación de los costos de varicela complicada (pacientes hospitalizados), en el Hospital Infantil Napoleón Franco Pareja de Cartagena, Colombia.

\section{MÉTODOS}

Se diseño un estudio de casos retrospectivo para evaluar los costos directos de hospitalización de varicela en una población pediátrica de la ciudad de Cartagena, Colombia. Para esto se tomaron todas las hospitalizaciones incurridas durante los años 2005 a 2008 en el Hospital Infantil Napoleón Franco Pareja (HINFP) en una población de 1 mes a 12 años cumplidos.

Procedimientos de recolección de información

Se dirigió la búsqueda inicialmente al Departamento de estadística del HINFP, donde se consultaron todos los casos de varicela (diagnósticos CIE10 B019, B018) 2005-2009. El estudio se realizó sobre los diagnósticos de egreso de varicela que se realizaron en el hospital. Posteriormente, y debido a la exactitud del conteo de los elementos utilizados en la atención hospitalaria por parte del hospital, se procedió al departamento financiero para extraer los costos incurridos.

Análisis de los costos

Todos los costos directos fueron extraídos desde la perspectiva del hospital. Los costos directos fueron categorizados en costos de atención (talento humano utilizado), costos de laboratorio (todos los exámenes de laboratorio), imagenología (rayos $\mathrm{X}$, tomografía axial computarizada, resonancia magnética), y medicamentos utilizados para la atención del paciente.

El microcosteo se realizó en pesos colombianos. Se realizó un ajuste por inflación de acuerdo al Índice de precios al consumidor del Departamento Administrativo Nacional de Estadística (DANE) (4). Los costos fueron expresados en pesos colombianos del 2010.

Todos los costos fueron expresados en medianas con rango intercuartílico (RIC) como medida de dispersión. Se compararon mediante test no paramétricos los costos en edades categorizadas ( $<1$ año, 1-5 años, y 5-12 
años). Un valor de $\mathrm{P}<0,05$ fue considerado significativo. Se analizaron gráficamente las diferencias entre los grupos mediante un box-plot.

\section{RESULTADOS}

En total se encontraron 40 casos de varicela hospitalizados y ambulatorios en el HINFP de 2005 a 2009, de los cuales se consiguieron 18 historias en pacientes hospitalizados. De los 40 pacientes, 25 (62,5\%) fueron del género masculino.

Dos pacientes presentaban tumores cancerosos (tumor de Wilms y tumor retroperitoneal).

La mediana de edad en los 18 pacientes pediátricos hospitalizados fue de 2,3 años (Q1: 0,6; Q3: 5,3). E1 33,3\% $(\mathrm{n}=6)$ de los pacientes hospitalizados fueron menores de 1 año, el 38,8 \% $(n=7)$ de 1-5 años, y 27,7 \% $(n=5)$ de 5 a 12 años. La mediana de casos presentados por año (2005-2009) fue de 3 (Q1: 2; Q3: 5). La mediana de estancia hospitalaria fue de 4 días (Q1: 1; Q3: 6; Mínimo: 1; Máximo: 13), con el 27,8 \% $(\mathrm{n}=5)$ de los pacientes con una estancia de 1 día o menos, el 44,4\% $(\mathrm{n}=8)$ con una estancia de 1-5 días, y el siguiente $27,7 \%(n=5)$ con más de 5 días en un hospital. En la Figura 1 se muestra el box-plot de días de estancia hospitalaria categorizado por edad.

\section{Costos totales}

La mediana de costos totales hospitalarios fue de \$ 898766 (Q1: \$ 197 348; Q3: \$ 1195 262). Los costos más altos en los que se incurrieron fueron consecuencia de la atención del personal de salud, con una mediana de costo de \$ 574364 (Q1: \$ 111 021; Q3: \$ 815 847). Lo menos costoso en todo el proceso de atención hospitalaria de estos pacientes pediátricos fue la imagenología (Tabla 1). La mediana de costo total por día fue de \$ 221 777 (Q1: \$ 97 027; Q3: \$ 293 740).

Costos categorizados por edad

Los costos categorizados por edad se encuentran en la Tabla 2. Ninguna de las comparaciones de edad con el test no paramétrico de Kruskal-Wallis fue 
significativa. La mediana de costos totales por día en $<1$ año fue de $\$ 231$ 218 (Q1: \$ 226 427; Q3: \$ 256508 ); en infantes de 1-5 años fue de \$ 217 127 (Q1: 70 610; Q3: 381 035); y en pacientes de 5-12 años de \$ 105833 (Q1: $39568 ;$ Q3: \$ 148 637). La Figura 2 presenta el box-plot de los costos por día de estancia hospitalaria categorizados por edad.

Figura 1. Box-plot de días de estancia hospitalaria en pacientes con varicela categorizada por grupos de edad

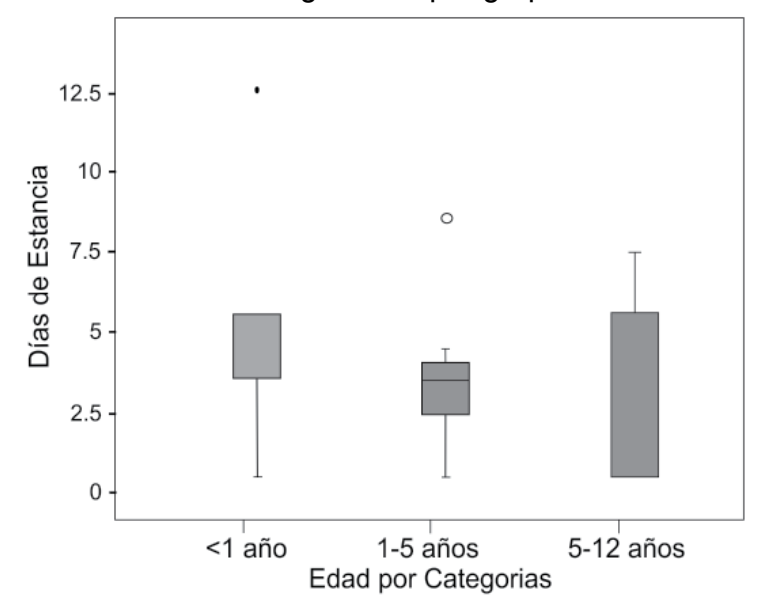

Tabla 1. Costos totales por paciente de varicela hospitalaria en pacientes pediátricos del HINFP (costos en pesos colombianos del 2010)

\begin{tabular}{lc}
\hline \multicolumn{1}{c}{ İtem } & $\begin{array}{c}\text { Costo Total } \\
\text { Mediana (RIC) }\end{array}$ \\
\hline Atención & $574364(111021-815$ 847) \\
Laboratorio & $43882(15989-177605)$ \\
Imagenología & $0(0-7$ 488) \\
Medicamentos & $95428(25338-440$ 977) \\
Costo Total & $898766(197348-1195262)$ \\
\hline
\end{tabular}


Tabla 2. Costos totales de varicela hospitalaria en pacientes pediátricos del HINFP categorizados por edad, con comparación mediante test no paramétrico (costos en pesos colombianos del 2010).

\begin{tabular}{|c|c|c|c|c|}
\hline Ítem & $\begin{array}{c}<1 \text { año } \\
\text { Mediana (RIC) }\end{array}$ & $\begin{array}{c}1-5 \text { años } \\
\text { Mediana (RIC) }\end{array}$ & $\begin{array}{c}\text { 5-12 años } \\
\text { Mediana (RIC) }\end{array}$ & $\begin{array}{l}\text { Valor } \\
\text { de P* }\end{array}$ \\
\hline Atención & $\begin{array}{c}693855 \\
(574364-823 \text { 348) }\end{array}$ & $\begin{array}{c}437583 \\
(189181-718692)\end{array}$ & $\begin{array}{c}34306 \\
(25833-772680)\end{array}$ & 0,371 \\
\hline Laboratorio & $\begin{array}{c}43882 \\
(16522-208994)\end{array}$ & $\begin{array}{c}123955 \\
(14390-183214)\end{array}$ & $\begin{array}{c}39147 \\
(17048-72855)\end{array}$ & 0,600 \\
\hline Imagenología & $\begin{array}{c}14976 \\
(0-41883)\end{array}$ & 0 & 0 & 0,119 \\
\hline Medicamentos & $\begin{array}{c}166080 \\
(68505-365902)\end{array}$ & $\begin{array}{c}191209 \\
(30668-666203)\end{array}$ & $\begin{array}{c}9348 \\
(0-32380)\end{array}$ & 0,170 \\
\hline Costo Total & $\begin{array}{c}980742 \\
(905708-1026 \\
031)\end{array}$ & $\begin{array}{c}793929 \\
(234239-1524 \\
139)\end{array}$ & $\begin{array}{c}105833 \\
(39568-891824)\end{array}$ & 0,274 \\
\hline
\end{tabular}

${ }^{*}$ Test de Kruskal-Wallis

Figura 2. Box-plot de costo hospitalario de varicela paciente/día, categorizada por grupos de edad en el HINFP

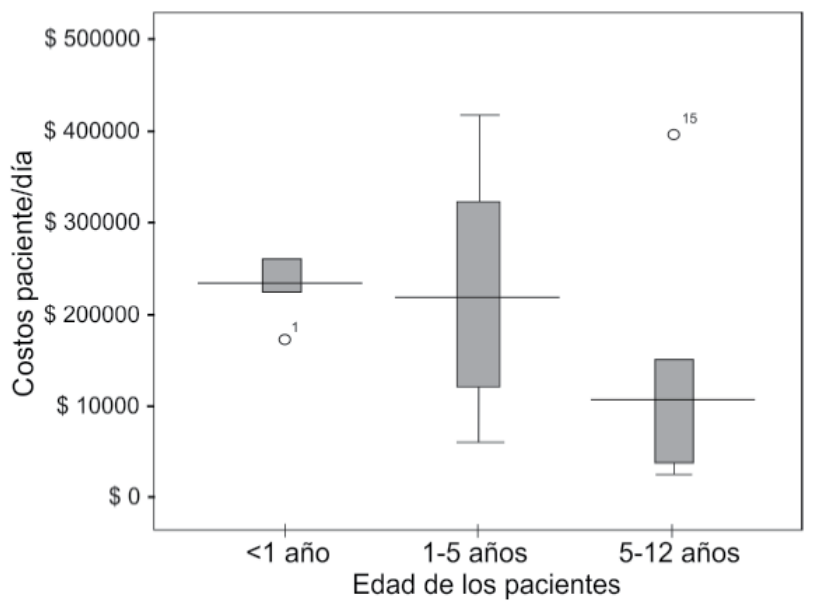

Nota: Un paciente (no mostrado) en el grupo de menores de 1 año (<1 año) tuvo un costo por día de estancia hospitalaria de \$1902 223.

\section{DISCUSIÓN}

Este es el primer estudio en Colombia que estima los costos de varicela en pacientes pediátricos hospitalizados. 
La varicela que necesita hospitalización se puede presentar en pacientes con comorbilidades asociadas que hacen al paciente susceptible de presentar una enfermedad severa, que complique el cuadro clínico del paciente $(3,5)$. Esta conjunción de patologías hace de la relativamente benigna varicela, una enfermedad potencialmente fatal.

La incidencia corregida de varicela en países desarrollados es del $5,2 \%$ de toda la población pediátrica (2). Esta incidencia acarrea costos significativos, tanto directos relacionados con la varicela, sus complicaciones y las comorbilidades asociadas; como costos ocasionados por pérdida de productividad en los padres o cuidadores de los pacientes.

En Canadá los costos medios por paciente de varicela no hospitalizada desde la perspectiva de la sociedad van desde \$236 dólares canadienses para niños en edad escolar (media de 6,3 años), hasta $\$ 370$ dólares canadienses para niños en guarderías (media de 2,8 años) (6). Para los casos hospitalizados por varicela, la media de costos por paciente son de $\$$ 7059 dólares canadienses (5).

Este estudio detalla los costos asociados con varicela complicada en Colombia. Como estudios previos han encontrado, los infantes más jóvenes son los que más costos relacionados presentan. Esto es especialmente cierto en niños menores de 1 año, con costos totales de atención de COP $\$ 980$ 742 (RIC: COP \$ 905 708-COP \$ 1026 031). En la revisión realizada por los autores, no se encontraron estudios de costos de varicela en países en desarrollo como Colombia (con producto interno bruto per cápita menor de USD \$ 12196 según el método Atlas), por lo que no se compararon medidas de costos con otros países.

Los costos de varicela fueron más altos, sin significancia estadística, en pacientes más menores de 1 año, comparados con los pacientes de 5 a 12 años. A pesar que no existe significancia estadística en las diferencias de los tres grupos, si se comparan los pacientes menores de un año, con los pacientes de 5 a 12 años, se describe un mayor costo, con una mediana de COP $\$ 980742$ (RIC: COP \$ 905708 -COP \$1 026 031) para menores de un año, y COP \$105 833 (RIC: COP \$39 568-COP \$ 891 824) para niños de 5 a 12 años. Esto permite sugerir mayores costos y gravedad de los pacientes menores de un año. 
En países desarrollados como Estados Unidos la vacunación universal contra varicela ha reducido mortalidad directa por varicela, y como causa contribuidora $(7,8)$.

A pesar de ser una enfermedad con una mortalidad baja (menos de 1 por millón/año en Estados Unidos) (9), las tasas de incidencia de la varicela, la carga de enfermedad que esto genera y los costos derivados, hacen que se considere la posibilidad de la implementación de la vacuna universal contra varicela, tanto en países desarrollados como en vías de desarrollo $(10-12)$.

Con la visión de introducir la vacuna en España, un estudio de costoefectividad de la vacuna contra varicela en adolecentes estimó que cada caso evitado de la enfermedad le costaría al sistema de salud $€ 131$, sin tener en cuenta la disminución de la incidencia del herpes zoster (11). En otro estudio español, la vacunación universal contra varicela, resultó costobeneficiosa en niños de 1 a 2 años (12).

Estos estudios previos en España y otros países (13-15), los costos en pacientes graves de Colombia, y la gravedad de los pacientes menores de un año, reportados en este estudio, permiten sugerir a los dirigentes de salud en Colombia el estudio de la introducción de la vacunación contra varicela en la población colombiana.

Este estudio tiene limitaciones. La principal limitación está relacionada con la poca cantidad de pacientes que se investigaron y lo limitado en la obtención de la muestra, pues solo se tomaron casos del HINFP con datos disponibles. Esto incide directamente tanto en la validez interna como en la generalización de los resultados a la población general. Sin embargo, los autores de este estudio consideran que dado que se extrapolarían a solo pacientes hospitalizados, lo aleatorio de la obtención muestra -la pérdida de datos no se relacionó con gravedad-, los 18 pacientes son un buen indicador de los costos en pacientes hospitalizados. Además, los resultados son coherentes con lo publicado previamente sobre el tema.

Estos resultados cimentan la evidencia a favor de la vacunación, e invitan a dirigentes en salud a considerar la introducción de la vacunación contra varicela en Colombia. Los costos derivados de la carga de enfermedad de varicela, una enfermedad infecciosa con una incidencia de un $5 \%$ en la 
población pediátrica; la mortalidad derivada de casos con comorbilidades graves; y la gravedad de estos casos en neonatos y menores de un año, hacen que sea imperante considerar la posibilidad de incluir la varicela en el plan ampliado de inmunizaciones -PAI- de Colombia

\section{REFERENCIAS}

1. Espinal CA, Upegu G, Cristancho LM. Indicadores epidemiológicos de varicela en Colombia. Infectio. 1998;2(2):77-85.

2. Nicolosi A, Sturkenboom M, Mannino S, Arpinelli F, Cantarutti L, Giaquinto C. The incidence of varicella: correction of a common error. Epidemiology. 2003 Jan;14(1):99-102.

3. Saez-Llorens X, De Suman O, De Moros D, Rubio Mdel P. Complications and costs associated with chickenpox in immunocompetent children. Rev Panam Salud Publica. 2002 Aug;12(2):111-6.

4. DANE. Indices de Precios al Consumidor.1996- 2011 [Internet]. Bogotá D.C.: Departamento Administrativo Nacional de Estadística; 2009; Available from: http://www.dane.gov. co/files/investigaciones/ipc/ene11/IPC_Variacion.xls. Consulted Enero 3, 2009.

5. Law B, Fitzsimon C, Ford-Jones L, McCormick J, Riviere M. Cost of chickenpox in Canada: part II. Cost of complicated cases and total economic impact. The Immunization Monitoring Program-Active (IMPACT). Pediatrics. 1999 Jul;104(1 Pt 1):7-14.

6. Law B, Fitzsimon C, Ford-Jones L, MacDonald N, Dery P, Vaudry W, et al. Cost of chickenpox in Canada: part I. Cost of uncomplicated cases. Pediatrics. 1999 Jul;104(1 Pt 1):1-6.

7. Giacchino R, Losurdo G, Castagnola E. Decline in mortality with varicella vaccination. N Engl J Med. 2005 Apr 28;352(17):1819.

8. Nguyen $\mathrm{HQ}$, Jumaan $A O$, Seward JF. Decline in mortality due to varicella after implementation of varicella vaccination in the United States. N Engl J Med. 2005 Feb 3;352(5):450-8.

9. Gil A, Oyaguez I, Carrasco P, Gonzalez A. Epidemiology of primary varicella hospitalizations in Spain. Vaccine. 2001 Nov 12;20(3-4):295-8.

10. Alonso J, Lozano de la Torre M, Madrigal V. Vacuna antivaricela: ¿llegó el momento de su inclusión en el calendario vacunal español? Bol Pediatr. 1999;39:229-35.

11. Pena-Rey I, Perez-Farinos N, Cortes-Garcia M, Amela-Heras C. Coste-efectividad de la vacunacion contra la varicela en adolescentes en Espana. Gac Sanit. 2004 JulAug;18(4):287-94.

12. Lenne X, Diez Domingo J, Gil A, Ridao M, Lluch JA, Dervaux B. Economic evaluation of varicella vaccination in Spain: results from a dynamic model. Vaccine. $2006 \mathrm{Nov}$ 17;24(47-48):6980-9.

13. Diez Domingo J, Ridao M, Latour J, Ballester A, Morant A. A cost benefit analysis of routine varicella vaccination in Spain. Vaccine. 1999 Mar 17;17(11-12):1306-11.

14. Coudeville L, Brunot $A$, Giaquinto $C$, Lucioni $C$, Dervaux $B$. Varicella vaccination in Italy : an economic evaluation of different scenarios. Pharmacoeconomics. 2004;22(13):839-55.

15. Coudeville L, Brunot A, Szucs TD, Dervaux B. The economic value of childhood varicella vaccination in France and Germany. Value Health. 2005 May-Jun;8(3):209-22. 